\title{
Transplantation for alcoholic liver disease: lessons from the explant?
}

\author{
A Bathgate, P Hayes
}

\section{Outcome of liver transplantation for patients with end stage alcoholic cirrhosis is not different from those with superimposed alcoholic hepatitis}

established, with good outcome in terms of patient and graft survival. Transplantation for alcoholic hepatitis however is not a recognised indication in the UK. ${ }^{1}$ All centres in the UK require a period of abstinence before listing for transplantation, although not all have input from psychiatrists experienced in alcohol dependence. ${ }^{2}$

The article by Tomé et al reports on the outcome of liver transplantation for alcoholic cirrhosis in a single centre from Spain that required abstinence for three months prior to transplantation. The explanted liver was examined for histological features of alcoholic hepatitisfocal necrosis, neutrophilic infiltrate, Mallory's hyaline degeneration, and liver steatosis. Patients with histological alcoholic hepatitis were compared with alcoholic cirrhotics without these features and found to have no significant outcome differences with respect to survival and recidivism.

The findings of alcoholic hepatitis on explant can be interpreted in two ways. The histological features had not yet disappeared following cessation of alcohol or patients were indeed continuing to drink while claiming abstinence. The period of abstinence given for the group with alcoholic hepatitis was a median of 15 months, which suggests that some patients were indeed drinking while claiming to be abstinent. However, some of those around the three month period of abstinence may well have had some recoverable liver function. Patients with alcoholic hepatitis on explant were more likely to have Child's C cirrhosis compared with those who did not. In both groups there appeared to be a sizeable number of patients with good liver function with one in six patients having Child's A cirrhosis. This is different to the experience in centres in the rest of Europe where at least $70 \%$ had Child's class $\mathrm{C}$ cirrhosis and none had Child's class $\mathrm{A}^{3}{ }^{4}$ It follows therefore that some patients may well have recovered liver function if alcoholic hepatitis had been allowed to recover fully. In our own centre the occurrence of alcoholic hepatitis in the explanted liver is extremely rare. We recommend a period of abstinence of

Tomé S, Martinez-Rey C, Gonzalez-Quintela A, et al. Influence of superimposed alcoholic hepatitis on the outcome of liver transplantation for end-stage alcoholic liver disease. J Hepatol 2002;36:793-8

Background: End stage alcoholic liver disease (ALD) is a common indication for liver transplantation but alcoholic hepatitis $(\mathrm{AH})$ is often regarded as a contraindication. There is little evidence to support this belief.

Objective: To study the effect of superimposed $\mathrm{AH}$ on the outcome of liver transplantation for end stage ALD.

Design: Case series of consecutive patients undergoing liver transplantation over a five year period at one centre, followed for a median of 30 months.

Patients: A total of 169 cases, 68 cases were due to ALD (all abstinent for at least three months). Over half of these $(n=36)$ had histological evidence of AH in the explanted liver. Their outcome was compared with 32 cases without hepatitis and also with the 101 cases undergoing transplant for other reasons, including hepatitis $\mathrm{C}$ virus (HCV) infection.

Results: Post transplant mortality was similar in all groups (one year survival: $72 \%, 81 \%$, and $81 \%$; three year survival: $68 \%, 75 \%$, and $75 \%$; five year survival: $61 \%, 75 \%$, and $50 \%$, for the ALD cirrhosis, ALD cirrhosis+AH, and HCV cirrhosis groups, respectively). In particular, superimposed $\mathrm{AH}$ did not adversely affect outcome. Recidivism occurred in 10\% of cases transplanted for ALD but was considered abusive in only two.

Conclusion: Survival after liver transplantation was similar in ALD cirrhosis patients with or without $\mathrm{AH}$ and cases transplanted for other reasons. Even histologically severe AH did not adversely affect outcome.

Points to note: Patients were allegedly abstinent and did not have clinically acute $\mathrm{AH}$, and hence are not representative of the acute cases we see in clinical practice. Therefore, conclusions cannot be drawn on the use of transplantation in these cases. We would like to broaden the discussion about this paper more generally to the issue of transplants for ALD-for example, in which ALD should the jobbing gastrointestinal physician consider referral for transplant?

six months, which is a compromise between the reported extremes of recoverable liver function-three months ${ }^{5}$ and 12 months. ${ }^{6}$

However, the major issue in liver transplantation for alcoholic liver disease is long term patient and graft survival. Recurrent disease is a very common situation in liver transplantation. Diseases such as hepatitis $C$ cirrhosis recur rapidly in approximately $20 \%$ of patients within five years. In this study, the $10 \%$ rate of recidivism did not differ between those patients with alcoholic hepatitis on explant and those without. The rate of recurrent disease was less than this, with $7 \%$ having histological evidence of recurrence and no graft losses. It would have been interesting to know if the two patients who drank excessively causing graft damage were in the group with alcoholic hepatitis but this is not made clear as there is evidence suggesting that steatosis and Mallory bodies in the explanted liver is a poor prognostic feature as regards recurrent alcoholic hepatitis and graft loss.

Acute alcoholic hepatitis, as described by Maddrey, defines a syndrome associated with hepatomegaly, neutrophilia, pyrexia, jaundice, and prolonged prothrombin time in the context of recent excessive alcohol intake. This syndrome often leads to renal failure and mortality is high. ${ }^{8}$ The clinical syndrome of acute alcoholic hepatitis is different from the histological alcoholic hepatitis presented in this study as the authors are careful to point out. Liver transplantation for acute alcoholic hepatitis cannot be substantiated on the basis of this study. This study does however highlight the excellent outcome of patients transplanted for alcoholic liver disease and that recurrent disease is relatively rare even in patients who may be economical with the truth at 
the time of assessment. In common with other centres, we recommend a policy of active involvement of psychiatrists in the transplant assessment and the application of a contract stipulating the patient's intention to remain abstinent. ${ }^{9}$

\section{Gut 2003;52:462-463}

\section{Authors' affiliation}

A Bathgate, P Hayes, Scottish Liver Transplant Unit, Royal Infirmary of Edinburgh, Edinburgh, UK

Correspondence to: Professor P Hayes: p.hayes@ed.ac.uk

\section{REFERENCES}

Neuberger J, Schulz K, Day C, et al. Transplantation for alcoholic liver disease. J Hepatol 2002;36:130-7.

2 Masterton G. Psychosocial factors in selection for liver transplantation. $B M$ 2000;320:263-4.

3 Mackie J, Groves K, Hoyle A, et al. Orthotopic liver transplantation for alcoholic liver disease: A retrospective analysis of survival, recidivism, and risk factors predisposing to recidivism. Liver Transpl 2001:7:418-27.

4 Poynard T, Barthelemy P, Fratte $S$, et al Evaluation of efficacy of liver transplantation in alcoholic cirrhosis by a case-control study and simulated controls. Lancet 1994;344:502-7.

5 Veldt BJ, Lainé F, Guillygomarc'h, et al. Indication of liver transplantation in severe alcoholic liver cirrhosis: quantitiative evaluation and optimal timing. $J$ Hepatol 2002;36:93-8

6 Mathurin P, Duchatelle V, Ramond M, et al. Survival and prognstic factors in paients with severe alcoholic hepatitits treated with prednisolone. Gastroenterology 1996;110:1848-53.

7 Conjeevaran HS, Hart J, Lissoos TW, et al Rapidly progressive liver injury and fatal alcoholic hepatitis occurring after liver transplantation in alcoholic patients. Transplantation 1999:67:1562-8.

8 Maddrey WC. Alcoholic hepatitis: clinicopathologic features and therapy. Semin Liv Dis 1988;8:91-102.

9 Gish R, Lee A, Brooks L, et al. Long-term follow-up of patients diagnosed with alcohol dependence or alcohol abuse who were evaluated for liver transplantation. Liver Transp/ $2001 ; 7: 581-7$.

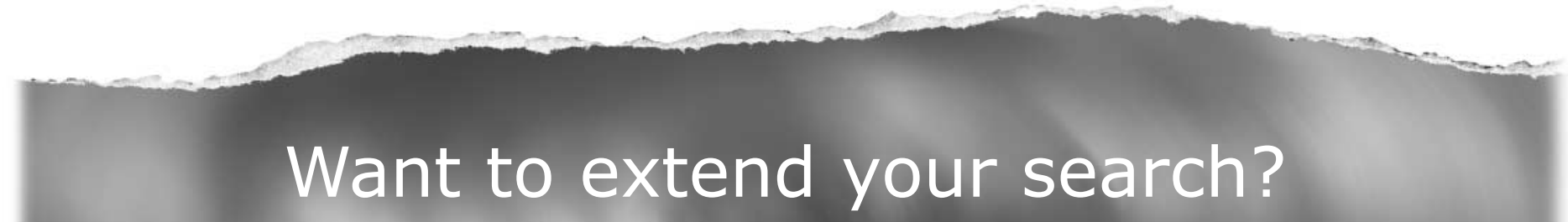

\section{Cross journal searching}

If you can't find what you are looking for in Gut you can extend your search across many of the more than 200 journals available for selection. You can restrict your search to specific subject areas

(eg, clinical medicine, basic research), or select specific journals, or search all available titles.

\section{www.gutjnl.com}

